\title{
Fractal Analysis in Liver Fibrosis
}

\section{To the Editor:}

We read with great interest the recent article by Moal et al. regarding application of fractal analysis to the differentiation of models of hepatic fibrosis. ${ }^{1}$ However, we have doubts about the linkage between fractal properties and the histologic appearance of liver specimens. Specifically, the only evidence of self-similarity for these liver specimens is their Fig. 3, i.e., the log-log plot from which the Kolmogorov fractal dimension was derived. In this particular example, the log-log plot, containing only 7 points covering less than one decade of box widths, is of convex shape. Moreover, the regression slope, if examined locally, varies from about -1.0 at the small box widths to about -2.0 at the large box widths, respectively. This means that the liver fibrosis specimens are not self-similar even for the narrow range of scale chosen by Moal et al. It also indicates that the fractal dimension computed using linear regression would depend strongly on the choice of data range. ${ }^{2}$

We provide our results of box counting analysis on two sets of data to account for the above observations. One image contains circles of identical size uniformly separated from each other, obviously a regular object that can be described completely using Euclidean geometry. The other is the Koch's triadic curve with fractal dimension 1.26. Fig. 1 shows that data obtained from the Koch curve fall exactly on one straight line for two orders of magnitude. In contrast, data from the uniformly spaced circles follow a convex shape, with local slope varying from -1.0 at small boxes to -2.0 at large boxes, respectively, strikingly similar to the appearance of Fig. 3 presented by Moal et al. If one analyzed the uniformly spaced circles using limited data range, e.g., between the two dashed lines in Fig. 1, the seemingly linear data behavior might falsely justify the legibility of linear regression to obtain a noninteger "fractal" dimension of 1.37. In reality, however, the object to be analyzed may not exhibit any fractal properties at all. In the case of liver fibrosis, we are afraid that Moal et al. may have used the box counting method in a similar erroneous manner.

This problem of "physical cutoff of data range" leading to confusing results has been shown previously, ${ }^{3}$ yet received little attention from the biomedical society. We have also shown that for networked structures, the "fractal" dimension analyzed by box counting is a statistical reflection of "pore size."4 In the two distinct models of hepatic fibrosis used by Moal et al., ${ }^{1}$ very likely it was the "spacing between fibrotic elements," rather than structural complexity, that happened to provide the morphometric differentiation between different models of cirrhosis.

It may be correct to state that biological objects exhibit selfsimilarity for only a limited range of scale (self-affine). But that is not to say that such biological objects are all subject to successful applications of fractal analysis using ruler scales covering less than a decade of range. The example of uniformly spaced circles with identical size is a counter-example that the noninteger "fractal" dimension can have nothing related to fractal properties. Hence, even if fractal analysis methods yield numeric values allowing quantitative comparisons in liver histopathology, the interpretation of results should best be exercised with cautions bearing the methodologic limitations in mind. 5

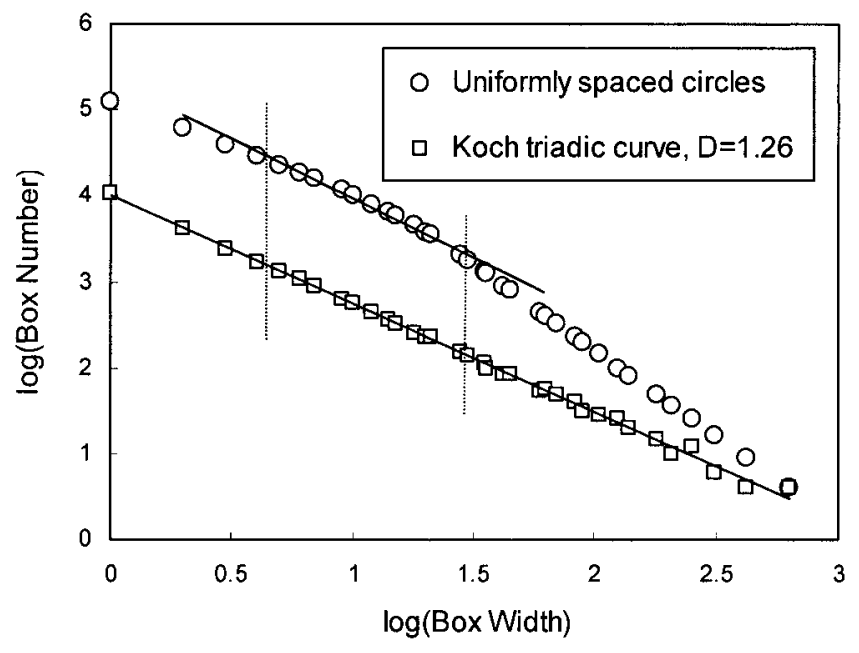

Fig. 1. Log-log plot shows box counting analysis on one nonfractal object (uniformly spaced circles of identical size; circles) and one ideal fractal object (Koch's triadic curve with fractal dimension 1.26; squares), from which the Kolmogorov fractal dimensions were derived. The data obtained from the Koch curve fall exactly on one straight line for at least two orders of magnitude, whereas the data from the nonfractal object follow a convex shape, with local slope varying from -1.0 at small boxes to -2.0 at large boxes, respectively, similar to the appearance of Fig. 3 in the article by Moal et al. Dashed lines show how a noninteger "fractal" dimension can be obtained for a nonfractal object if one analyzed the object using limited range of data covering less than one decade of ruler scale.

\author{
HSiaO-Jen Chung, M.D. \\ Division of Urology \\ Department of Surgery \\ Veterans General Hospital \\ Taipei, Taiwan, R.O.C. \\ Institute of Clinical Medicine \\ National Yang-Ming University, \\ Taipei, Taiwan, R.O.C.
}

\section{References}

1. Moal F, Chappard D, Wang J, Vuillemin E, Michalak-Provost S, Rousselet MC, Oberti F, et al. Fractal dimension can distinguish models and pharmacologic changes in liver fibrosis in rats. HePATOLOGY 2002;36:840-849.

2. Chung HW, Chung HJ. Correspondence re: J. W. Baish and R. K. Jain, Fractals and cancer. Cancer Res, 60:3683-3688, 2000. Cancer Res 2001; 61:8347-8350

3. Ciccotti M, Mulargia F. Pernicious effect of physical cutoffs in fractal analysis. Phys Rev E 2002;65:037201.

4. Chung HW, Chu CC, Underweiser M, Wehrli FW. On the fractal nature of trabecular structure. Med Phys 1994;21:1535-1540.

5. Avnir D, Biham O, Lidar D, Malcai O. Is the geometry of nature fractal? Science 1998;279:39-40.

Copyright (C) 2003 by the American Association for the Study of Liver Diseases. doi:10.1053/jhep.2003.50046

Wen-Chau Wu, B.S.

Hsiao-Wen Chung, Ph.D.

Department of Electrical Engineering

National Taiwan University

Taipei, Taiwan, R.O.C. 